\section{Dynamic Versus Static Weighting of Lyapunov Functions}

\author{
L. Praly, D. Carnevale, and A. Astolfi
}

\begin{abstract}
The relation between static and dynamic Lyapunov functions weighting is discussed. It is shown that, under some technical assumptions, stabilizability by means of static weighting implies stabilizability by means of dynamic weighting. The existence result is illustrated by means of an example which highlights that the design based on dynamic weighting requires less a-priori information on the system to be stabilized.
\end{abstract}

Index Terms - Lyapunov function, nonlinear.

\section{INTRODUCTION}

Lyapunov function scaling is a well-established analysis and design tool in nonlinear control design. It has been used, for example, to establish a Lyapunov of the reduction principle arising in center manifold theory [2], [3], in the study of stability properties of interconnected systems [4]-[10], in the design of stabilizing control laws for cascaded or feedback interconnected systems [11], [12], and in adaptive control systems [13]-[16]. Informally, the idea of Lyapunov function scaling can be described as follows. Consider a (nonlinear) system, and two functions $V_{1}$ and $V_{2}$ such that the time derivatives of each of these functions, along the solutions of the system, are nonpositive on some sets of the state space, the union of which coincide with the whole state space. Lyapunov function scaling allows to determine, if possible, scaling functions $l_{1}$ and $l_{2}$ such that the function $l_{1}\left(V_{1}\right)+l_{2}\left(V_{2}\right)$ is positive definite (and radially unbounded) and its time derivative is nonpositive in the whole state space. A second well-established analysis and design tool is dynamic scaling. Dynamic scaling essentially consists in adding a state component used as a scaling factor. This scaling factor could play the role of a state norm observer, see [17], [18]. As such it has been exploited in adaptive control, to render the boundedness property robust, see for instance [19] for nonlinear adaptive control, in nonlinear stabilization, to cope with input disturbances, see [20], and in nonlinear observers, to deal with non-Lipschitz nonlinearities [21]. Alternatively, it could be used to estimate the local incremental rate of a dynamical system. As such it is helpful in output feedback stabilization, see, for instance, [22] or [23]. By merging the above two tools, Lyapunov-like functions defined as sums of dynamically weighted partial Lyapunov functions can be constructed. Preliminary results using this technique are reported in [16] for the case of observer design and adaptive control and in [24] for the stabilization of simple cascades.

The techincal note is organized as follows. In Section II, an introductory example and the objectives of the techincal note are presented.

Manuscript received January 10, 2012; revised July 01, 2012, July 03, 2012, July 30, 2012; accepted October 06, 2012. Date of publication November 22, 2012; date of current version May 20, 2013. This work was supported in part by the PRIN project and ENEA-Euratom. Recommended by Associate Editor L. Marconi.

L. Praly is with MINES ParisTech, CAS, Mathématiques et Systèmes, Fontainebleau, France (e-mail: Laurent.Praly@minesparistech.fr).

D. Carnevale is with the Dipartimento di Informatica, Sistemi e Produzione, Università di Roma Tor Vergata, Rome 00133, Italy (e-mail: carnevale@disp. uniroma2.it).

A. Astolfi is with Department of Electrical and Electronic Engineering, Imperial College London, London SW7 2AZ, U.K. and with the Dipartimento di Informatica, Sistemi e Produzione, Università di Roma Tor Vergata, Rome 00133, Italy (e-mail: a.astolfi@imperial.ac.uk).

Digital Object Identifier 10.1109/TAC.2012.2229813
Section III contains the main results of the techincal note, namely a technical lemma followed by two formal statements establishing, under some technical conditions, the strong link between static weighting and dynamic weighting. Conclusions are given in Section IV.

\section{AN InTROdUCtORY EXAMPLE AND GOAL OF THE PAPER}

To illustrate the underlying ideas of static and dynamic Lyapunov function weighting we consider the problem of studying the stability properties of a simple cascade. To this end, consider the nonlinear system

$$
\dot{z}=-z+z y, \quad \dot{y}=-y
$$

and note that a simple analysis allows to conclude that the origin is a globally asymptotically stable equilibrium. To establish this stability result by means of a Lyapunov function, following [4], for instance, consider the two functions $V_{1}(y)=y^{2}$ and $V_{2}(z)=z^{2}$, two weighting functions $\ell_{1}$ and $\ell_{2}$, and the Lyapunov function candidate $V(y, z)=l_{1}\left(V_{1}(y)\right)+l_{2}\left(V_{2}(z)\right)$. Since $(1 / 2) \dot{V}=-\ell_{1}^{\prime}\left(V_{1}(y)\right) y^{2}-\ell_{2}^{\prime}\left(V_{2}(z)\right) z^{2}+\ell_{2}^{\prime}\left(V_{2}(z)\right) z^{2} y, \dot{V}$ is negative definite if the functions $\ell_{1}$ and $\ell_{2}$ are chosen to satisfy the conditions

$$
\frac{\ell_{1}^{\prime}\left(y^{2}\right)}{\ell_{2}^{\prime}\left(z^{2}\right)}>\frac{z^{2} \max \{0,|y|-1\}}{y^{2}}
$$

or, alternatively, the conditions $\ell_{1}^{\prime}\left(y^{2}\right) \geq 1 \geq\left(\max \{0,|y|-1\} / y^{2}\right)$, and $\ell_{2}^{\prime}\left(z^{2}\right) \leq\left(1 / 1+z^{2}\right) \leq\left(1 / z^{2}\right)$, for all $y$ and $z$. The above conditions yield the Lyapunov function $V(y, z)=y^{2}+\log \left(1+z^{2}\right)$, which is such that $\dot{V}<0$ for all nonzero $(y, z)$. An alternative way to study the properties of the solutions of system (1) is by means of dynamic Lyapunov function weighting. Following the arguments in [16], consider the Lyapunov-like function ${ }^{1} V_{r}(z, y)=V_{1}(y)+(1 / r) V_{2}(z)$, where $r(t) \geq r_{\star}>0$ for all $t \geq 0$, is the weighting variable. The time derivative of the Lyapunov-like functions along the trajectories of the system is

$$
\frac{1}{2} \dot{V}_{r}=-y^{2}-\frac{z^{2}}{r}+\frac{z^{2} y}{r}-\frac{z^{2}}{r} \frac{\dot{r}}{2 r}
$$

hence selecting

$$
\frac{\dot{r}}{r}=\frac{1}{2}+y^{2}-\frac{r-r_{\star}}{r}
$$

with $r(0) \geq r_{\star}$, yields $r(t) \geq r_{\star}$ and

$$
\frac{1}{2} \dot{V}_{r} \leq-y^{2}-\frac{z^{2}}{r}+\frac{z^{2}}{2 r} \frac{r-r_{\star}}{r} \leq-y^{2}-\frac{z^{2}}{2 r} .
$$

As a result, $z(t) / \sqrt{r(t)} \in L_{\infty}, y(t) \in L_{1}$. Note, however, that we cannot draw any conclusion on the properties of the zero equilibrium of the system, since no property of the behavior of $r$ has been established. One way to complete the analysis is via the (true) Lyapunov function, on $\mathbb{R} \times \mathbb{R} \times \mathbb{R}_{+*}{ }^{2}, \mathcal{U}(y, z, r)=V_{r}+\int_{2 r_{\star}}^{r} \operatorname{sat}\left(s-2 r_{\star}\right) / s d s$, the time derivative of which, along the trajectories of the system, satisfies the inequality

$$
\dot{\mathcal{U}} \leq-\left(y^{2}+\frac{z^{2}}{r}+\frac{\left(r-2 r_{\star}\right) \operatorname{sat}\left(r-2 r_{\star}\right)}{2 r}\right) .
$$

\footnotetext{
${ }^{1}$ This is not a Lyapunov function per se, since it is not positive definite and radially unbounded in $(y, z, r)$.

${ }^{2} \mathbb{R}_{+*}$ denotes the set of strictly positive real numbers.
} 
As a result, the point $\left(0.0,2 r_{\star}\right)$ is an (locally exponentially) asymptotically stable equilibrium point with domain of attraction $\mathbb{R} \times \mathbb{R} \times \mathbb{R}_{+*}$. Although the considered system has state dimension 2, the analysis above is carried out for a system with dimension 3 but admitting the given system as a subsystem. It follows that any stability property of the extended system can be projected onto the given system. The analysis by means of the dynamically weighted Lyapunov function presents a few advantages and disadvantages that are worth pointing out.

1) The dynamically weighted Lyapunov function is (trivially) constructed as a linear combination of the two functions $V_{1}$ and $V_{2}$ with a coefficient which depends upon the weighting variable $r$. On the other hand, the dynamic of the weighting variable may be hard to select.

2) Boundedness of the weighting variable $r$ is established a-posteriori.

3) There is no clear relation between the statically weighted Lyapunov function and the dynamically weighted one, i.e. between the constraint (2) on the ratio $\ell_{1}^{\prime}\left(V_{1}\right) / \ell_{2}^{\prime}\left(V_{2}\right)$ and the expression of $\dot{r}$ in (3). In particular, existence of one does not imply, in general, existence of the other.

We conclude the section noting that in the simple, motivating, example discussed above we have focused on stability analysis, while in the rest of the techincal note we deal with a feedback design problem in a general context. More specifically, aim of this techincal note is to partly address the issues raised at the end of Section II. In particular, a technical result, establishing a link between statically weighted control Lyapunov functions and dynamically weighted control Lyapunov functions is presented. This result gives conditions under which, with an additional technical assumption for each case, both weighted control-Lyapunov function and dynamically weighted Lyapunov function exist.

\section{MAIN RESULTS}

\section{A. A Technical Lemma}

Consider a nonlinear system described by equations of the form

$$
\dot{x}=f(x)+g(x) u
$$

with state $x(t) \in \mathbb{R}^{n}$, input $u(t) \in \mathbb{R}^{m}$, and, without loss of generality, $f(0)=0$.

Assume that there exist three functions $V_{1}: \mathbb{R}^{n} \rightarrow \mathbb{R}_{+}, V_{2}: \mathbb{R}^{n} \rightarrow$ $\mathbb{R}_{+}$and $R: \mathbb{R}^{n} \rightarrow\left[r_{0},+\infty\right)$, with $r_{0}>0$, such that the following holds.

(P1) The function $V_{1}+V_{2}$ is positive definite and radially unbounded.

(P2) For each pair $(x, r)$ satisfying $x \neq 0, \quad r \geq$ $R(x), \quad r L_{g} V_{1}(x)+L_{g} V_{2}(x)=0$ the inequality $r L_{f} V_{1}(x)+L_{f} V_{2}(x)<0$ holds.

(P3) For each strictly positive real number $\varepsilon$ there exists a strictly positive real number $\delta$ such that, for each pair $(x, r)$ satisfying $|x| \leq \delta, r \geq r_{0}, r L_{g} V_{1}(x)+L_{g} V_{2}(x) \neq 0$, and

$$
\frac{r L_{f} V_{1}(x)+L_{f} V_{2}(x)}{r L_{g} V_{1}(x)+L_{g} V_{2}(x)}<\varepsilon .
$$

Under assumption (P1), assumptions (P2) and (P3) are stating that, for $r$ fixed to a sufficiently large positive value, the function $x \mapsto V_{1}(x)+$ $(1 / r) V_{2}(x)$ is a Control Lyapunov Function (CLF) satisfying the Small Control Property (SCP) [25].

Remark 1: In the sequel we shall see that $R$ in (P2) is the key ingredient to design the weights of the statically weighted control-Lyapunov function and to design the update law of the weighting factor of the dynamically weighted one. Specifically, the weights $\ell_{1}$ and $\ell_{2}$ should be such that $\ell_{1}^{\prime}\left(V_{1}(x)\right) / \ell_{2}^{\prime}\left(V_{2}(x)\right) \geq R(x)$ and, similarly, $R(x(t))$ is what $r(t)$ should be.
Remark 2: Without the knowledge of $R$ one could try to define $\dot{r}$ indirectly, that is not from what it should be, but from the properties that it allows to achieve. For example, $\dot{r}$ may be such that, when $r$ is large enough, a function $h$ of the state is integrable along closed-loop solutions. This selection yields, for $r$ large, the update law $\dot{r}=h(x)$, which however may lead to severe nonrobustness problems, since arbitrarily small perturbations or the presence of noise may prevent $h(x(t))$ from converging to zero sufficiently fast. This may not be an issue in analysis problems but it is certainly one in design problems. We are now ready to establish a preliminary result.

Lemma 1: Consider system (5). Assume conditions (P1) to (P3) hold. Then there exists a function $\phi$ defined and continuous in the set $\{(x, r): r \geq R(x)\}$ satisfying

$$
\begin{aligned}
\bar{W}(x, r)=L_{f} V_{1}(x) & +\frac{1}{r} L_{f} V_{2}(x) \\
& +\left(L_{g} V_{1}(x)+\frac{1}{r} L_{g} V_{2}(x)\right) \phi(x, r)<0
\end{aligned}
$$

for all $(x, r)$ such that $x \neq 0$ and $r \geq R(x)$.

Lemma 1 is a direct consequence of what is known on universal formulae for the design of state feedback laws exploiting CLFs satisfying the SCP, see [25], [26].

Remark 3: The reader should not be misled by the result in Lemma 1 , which does not establish that $\phi$ is a stabilizing state feedback. Indeed, the expression on the 1.h.s. of the inequality (6) is the time derivative of the weighted Lyapunov function $V_{1}+V_{2} / r$ for $r$ constant, whereas inequality (6) holds only provided $r$ is larger than $R(x)$. Hence, if $R$ is a bounded function, a stabilizer from $\phi$ is obtained selecting $r \geq$ $\sup _{x} R(x)$ whereas, if $R$ is unbounded, either we consider only compact sets and obtain semi-global asymptotic stability, or we allow $r$ to follow the variations of $R(x)$. This latter case has to be dealt with care. In fact the function $x \mapsto \phi(x, R(x))$ is, in general, not a stabilizer since $x \mapsto V_{1}(x)+V_{2}(x) / R(x)$ may not be a CLF.

\section{B. Static Weighting}

Consider system (5) and the problem of designing a static state feedback

$$
u=\varphi(x)
$$

such that the origin of the closed-loop system is asymptotically stable. This problem admits a solution if conditions (P1) to (P3) hold and provided an additional technical assumption is satisfied by the triple $\left(V_{1}, V_{2}, R\right)$.

Proposition 1: Assume conditions (P1) to (P3) hold. If the triple $\left(V_{1}, V_{2}, R\right)$ is such that there exists a pair $\left(\ell_{1}, \ell_{2}\right)$ of $C^{1}$, class $\mathcal{K}^{\infty}$ functions, with nowhere zero derivative, satisfying

$$
\ell_{1}^{\prime}\left(V_{1}(x)\right) \geq R(x) \ell_{2}^{\prime}\left(V_{2}(x)\right) \quad \forall x \in \mathbb{R}^{n}
$$

then there exists a continuous functions $\varphi$ such that the origin is an asymptotically stable equilibrium of the closed-loop system (5)-(7).

Proof: Let $V_{\ell}(x)=\ell_{1}\left(V_{1}(x)\right)+\ell_{2}\left(V_{2}(x)\right)$. From the properties of $\ell_{1}$ and $\ell_{2}$, and condition (P1), this function is positive definite and radially unbounded. Consider now any continuous function $\phi$ defined on the set $\{(x, r): r \geq R(x)\}$ and satisfying inequality (6), and define the feedback (7) as $\varphi(x)=\phi\left(x, \ell_{1}^{\prime}\left(V_{1}(x)\right) / \ell_{2}^{\prime}\left(V_{2}(x)\right)\right)$. Since $\ell_{1}^{\prime}$ and $\ell_{2}^{\prime}$ have nonzero values and (8) holds, this function is well-defined and continuous on $\mathbb{R}^{n}$ and

$$
\begin{aligned}
W_{\ell}(x)=\bar{W} & \left(x, \frac{\ell_{1}^{\prime}\left(V_{1}(x)\right)}{\ell_{2}^{\prime}\left(V_{2}(x)\right)}\right)=L_{f} V_{1}(x)+\frac{\ell_{2}^{\prime}\left(V_{2}(x)\right)}{\ell_{1}^{\prime}\left(V_{1}(x)\right)} \\
& \times L_{f} V_{2}(x)+\left(L_{g} V_{1}(x)+\frac{\ell_{2}^{\prime}\left(V_{2}(x)\right)}{\ell_{1}^{\prime}\left(V_{1}(x)\right)} L_{g} V_{2}(x)\right) \varphi(x)
\end{aligned}
$$


is, by Lemma 1 and (8), negative definite. The claim thus follows since, along the solutions of the closed-loop system, $\overbrace{V_{\ell}(x)}=\ell_{1}^{\prime}\left(V_{1}(x)\right) W_{\ell}(x)$.

\section{Dynamic Weighting}

Consider system (5) and the problem of designing a dynamic state feedback

$$
\dot{r}=\psi(x, r), \quad u=\varphi(x, r)
$$

such that the closed-loop system (5), (9) has the following properties:

- the signal $r(t)$ remains in some compact subset of $\left[r_{0},+\infty\right)$ for all $t \geq 0$;

- there exists some nominal value $r_{\star} \geq r_{0}$ such that the point $(x, r)=\left(0, r_{\star}\right)$ is a globally stable equilibrium;

- the $x$ component converges to zero as time goes to infinity. As expressed in the following statement, this problem admits a solution if conditions (P1) to (P3) hold and provided an additional technical assumption is satisfied by the triple $\left(V_{1}, V_{2}, R\right)$.

Proposition 2: Assume conditions (P1) to (P3) hold. If the triple $\left(V_{1}, V_{2}, R\right)$ is such that the function $V_{1}+(1 / R) V_{2}$ is radially unbounded then there exist continuous functions $\varphi$ and $\psi$ and a constant $r_{\star}>R(0)$ such that the closed-loop system (5)-(9) has the following properties.

- The set $\mathbb{R}^{n} \times(R(0),+\infty)$ is forward invariant.

- $\left(0, r_{\star}\right)$ is a stable equilibrium.

- For each initial condition $(x, r)$ in $\mathbb{R}^{n} \times(R(0),+\infty)$, the $x$ component converges to zero as time goes to infinity.

Proof: To begin with observe that since the function $V_{2}$ takes only nonnegative values, zero can only be a minimum and therefore a critical value of $V_{2}$. It follows that

$$
\left\{x \in \mathbb{R}^{n} \mid V_{2}(x)=0\right\} \subseteq\left\{x \in \mathbb{R}^{n} \mid \frac{\partial V_{2}}{\partial x}(x)=0\right\} .
$$

Consider now the function $V_{r}(x)=V_{1}(x)+(1 / r) V_{2}(x)$, which, as a function of $(x, r)$, is defined and $C^{1}$ on $\mathbb{R}^{n} \times \mathbb{R}_{+*}$, and it is such that, by condition (P1), $\left\{r \geq r_{0}, V_{r}(x)=0\right\} \Longrightarrow x=0$ and, for all real numbers $\bar{r} \geq r_{0}$ and $\bar{v}$, there exists a real number $\bar{\xi}$ such that $V_{r}(x)>\bar{v}$ for all $(x, r):|x| \geq \bar{\xi}, r \in\left[r_{0}, \bar{r}\right]$. In words, this says that $x \mapsto V_{r}(x)$ is a positive definite and radially unbounded function, uniformly in $r$, in compact subsets of $\left[r_{0},+\infty\right)$.

Select any continuous function $\phi$, defined on the set $\{(x, r): r \geq$ $R(x)\}$ and satisfying inequality (6). Note that Lemma 1 guarantees the existence of such a function. Define in addition the function $\varphi$ of the feedback (9) as

$$
\varphi(x, r)=\phi(x, \max \{r, R(x)\})
$$

and the function $W(x, r)=\bar{W}(x, \max \{r, R(x)\})$ as

$$
\begin{aligned}
W(x, r)= & L_{f} V_{1}(x)+\frac{1}{\max \{r, R(x)\}} L_{f} V_{2}(x) \\
& +\left(L_{g} V_{1}(x)+\frac{1}{\max \{r, R(x)\}} L_{g} V_{2}(x)\right) \varphi(x, r) .
\end{aligned}
$$

Along the solutions of the closed-loop system we have

$$
\begin{aligned}
\overbrace{V_{r}(x)}=W(x, r)+ & {\left[L_{f} V_{2}(x)+L_{g} V_{2}(x) \varphi(x, r)\right] } \\
& \times \frac{\max \{R(x)-r, 0\}}{r R(x)}-\frac{V_{2}(x)}{r^{2}} \psi(x, r) .
\end{aligned}
$$

By Lemma $1, W(x, r)$ is strictly negative for all nonzero $x$ and all $r$, hence (10) implies that $\overbrace{V_{1}(x)+V_{2}(x) / r}<0, \forall(x, r): r>$ $0, V_{2}(x)=0$. As a result, $x \mapsto V_{1}(x)+(1 / r) V_{2}(x)$ is a partial (not in $r$ ) CLF for the interconnected system (5)-(9), with $\varphi$ as in (11) and $\psi$ as control input, for which the SCP holds trivially for $r>R(0)$.

From this property, we look for a $\psi$, possibly rendering the set $\left\{(x, r): r \geq r_{\star}\right\}$, with $r_{\star}>R(0)$, forward invariant along the solutions of the closed-loop system, to achieve the stabilization objective. To this end, define ${ }^{3}$

$$
\tilde{\psi}= \begin{cases}0, & \text { if } V_{2}(x)=0, \\ \frac{\max \left\{\alpha W+\left[L_{f} V_{2}+L_{g} V_{2} \varphi\right] \frac{\max \{R-r, 0\}}{r R}, 0\right\} r^{2}}{V_{2}}, & \text { elsewhere }\end{cases}
$$

with $\alpha \in(0,1)$. By classical results on CLF, see [25]-[27], the function $\tilde{\psi}$ is well-defined and continuous on $\mathbb{R}^{n} \times(R(0),+\infty)$ and it is such that $\forall(x, r): r>R(0)$ setting $\psi=\tilde{\psi}$ yields

$$
\overbrace{\frac{V_{1}(x)+V_{2}(x)}{r}}^{i} \leq(1-\alpha) W(x, r) .
$$

Note that $\tilde{\psi}$ takes only nonnegative values, hence selecting $\psi=\tilde{\psi}$ yields a monotonically nondecreasing $r$ along the solutions of the closed-loop system. This problem can be solved recalling that the function $W$ takes nonpositive values and selecting

$$
\psi(x, r)=\tilde{\psi}(x, r)+\frac{r \beta W(x, r)}{1+V_{2}(x)} \max \left\{r-r_{\star}, 0\right\}
$$

with $\beta \in(0,1-\alpha)$. In fact, since $\left(r / 1+V_{2}(x)\right) \max \{r-$ $\left.r_{\star}, 0\right\}\left(V_{2}(x) / r^{2}\right) \leq 1$, by (13) and (15), this selection yields

$$
\overbrace{\frac{V_{1}(x)+V_{2}(x)}{r}}^{r} \leq(1-\alpha-\beta) W(x, r) .
$$

We conclude that, on the positive time domain of existence of closedloop solutions, $V_{1}(x)+(1 / r) V_{2}(x)$ is bounded and $W(x, r)$ is integrable. To complete the analysis of the solutions of the closed-loop system observe that given a $C^{1}$, class $\mathcal{K}^{\infty}$ function $\rho_{1}$, to be defined, with derivative $\rho_{1}^{\prime}$ of class $\mathcal{K}^{\infty}$, there exists a class $\mathcal{K}^{\infty}$ function $\mu$ such that ${ }^{4}$, for any pair $(a, b)$ of strictly positive real numbers

$$
-a+b \leq \frac{\rho_{1}(b)}{\mu(a)} .
$$

As a result, recalling that $W$ is nonpositive and that $\max \{R(x)-$ $r, 0\} / R(x)$ takes values in $[0,1]$, then from (14) it holds

$$
\tilde{\psi} \leq \frac{\rho_{1}\left(\left|L_{f} V_{2}+L_{g} V_{2} \varphi\right|\right)}{\mu(\alpha|W|)} \frac{\max \{R-r, 0\}}{R} \frac{r}{V_{2}}
$$

${ }^{3}$ For compactness, in what follows we drop the arguments of the functions whenever this does not cause confusion.

${ }^{4}$ As suggested by Reviewers a possible choice is $\mu(a)=\rho_{1}^{\prime}(a)$.

$$
\begin{aligned}
\overbrace{\mathcal{U}(x, r)} & \leq(1-\alpha-\beta) W(x, r) \kappa^{\prime}\left(V_{1}(x)+\frac{V_{2}(x)}{r}\right)+\gamma \frac{\max \left\{r-r_{\star}, 0\right\}\left(\tilde{\psi}(x, r)+\beta W(x, r) \frac{r}{1+V_{2}(x)} \max \left\{r-r_{\star}, 0\right\}\right)}{\sqrt{1+\max \left\{r-r_{\star}, 0\right\}^{2}}} \\
& \leq(1-\alpha-\beta-\gamma) W(x, r) \kappa^{\prime}\left(V_{1}(x)+\frac{V_{2}(x)}{r}\right)+\gamma \beta W(x, r) \frac{r}{1+V_{2}(x)} \frac{\max \left\{r-r_{\star}, 0\right\}^{2}}{\sqrt{1+\max \left\{r-r_{\star}, 0\right\}^{2}}} .
\end{aligned}
$$


for all $(x, r): r>0, V_{2}(x) \neq 0$. On the other hand (10) implies $L_{f} V_{2}(x)+L_{g} V_{2}(x) \varphi(x, r)=0$ for all $(x, r): V_{2}(x)=$ 0 and therefore, by continuity, $\max _{r \in\left[r_{0}, R(x)\right]}\left\{L_{f} V_{2}(x)+\right.$ $\left.L_{g} V_{2}(x) \varphi(x, r)\right\}=0 \forall x: V_{2}(x)=0$. Note also that the function $x \mapsto \max _{r \in\left[r_{0}, R(x)\right]}\left\{L_{f} V_{2}(x)+L_{g} V_{2}(x) \varphi(x, r)\right\}$ is continuous. It follows that there exist class $\mathcal{K}^{\infty}$ functions $\rho_{1}$ and $\rho_{2}$ such that $\rho_{1}$ is $C^{1}$, its derivative $\rho_{1}^{\prime}$ is a class $\mathcal{K}^{\infty}$ function and

$$
\rho_{1}\left(\left|L_{f} V_{2}(x)+L_{g} V_{2}(x) \varphi(x, r)\right|\right) \leq V_{2}(x)\left[1+\rho_{2}(|x|)\right]
$$

for all $(x, r): r \in\left[r_{0}, R(x)\right]$, yielding

$$
\tilde{\psi}(x, r) \leq \frac{r\left[1+\rho_{2}(|x|)\right]}{\mu(\alpha|W(x, r)|)} \frac{\max \{R(x)-r, 0\}}{R(x)}
$$

$\forall(x, r): r \geq r_{0}, V_{2}(x) \neq 0$. Observe now that to any $r_{\star}>R(0)$ we can associate a neighborhood $\mathcal{N}$ of the origin such that $\max \{R(x)-$ $r, 0\}=0$ for all $x \in \mathcal{N}$ and all $r \geq r_{\star}$. In addition $W(x, r)$ is strictly negative for all $x \notin \mathcal{N}$ and all $r \geq r_{\star}$. Moreover, since $\max \{R(x)-$ $r, 0\}=0$, when $r \geq R(x)$, and the function $V_{1}+(1 / R) V_{2}$ is radially unbounded, there exists a $C^{1}$ function $\kappa: \mathbb{R}_{+} \rightarrow \mathbb{R}_{+}$, with nondecreasing and nonintegrable derivative $\kappa^{\prime}$, satisfying

$$
\begin{aligned}
& r\left[1+\rho_{2}(|x|)\right] \frac{\max \{R(x)-r, 0\}}{R(x)} \\
& \quad \leq \mu(\alpha|W(x, r)|)|W(x, r)| \kappa^{\prime}\left(V_{1}(x)+\frac{V_{2}(x)}{r}\right)
\end{aligned}
$$

for all $x$ and all $r \geq r_{\star}$. In conclusion, the bound

$$
\tilde{\psi}(x, r) \leq W(x, r) \kappa^{\prime}\left(\frac{V_{1}(x)+V_{2}(x)}{r}\right)
$$

$\forall(x, r): r \geq r_{\star}, V_{2}(x) \neq 0$ holds. Consider now the function

$\mathcal{U}(x, r)=\kappa\left(V_{1}(x)+\frac{V_{2}(x)}{r}\right)+\left(\sqrt{1+\max \left\{r-r_{\star}, 0\right\}^{2}}-1\right)$

with $\gamma \in(0,1-\alpha-\beta)$. With (16), exploiting the inequalities (17) and (21) yields (see the equation at the bottom of the previous page). This inequality, together with inequality (17), establishes the result.

Remark 4: If a continuous function $\varpi: \mathbb{R}^{n} \times(0,+\infty) \rightarrow \mathbb{R}_{+*}$ satisfying $V_{2}(x) \varpi(x, r) \leq r W(x, r)$, for all $(x, r): r \geq r_{\star}$ is known, then we can replace $\psi$ in (16) with $\psi(x, r)=\tilde{\psi}(x, r)-\beta \varpi(x, r)(r-$ $\left.r_{\star}\right)$. Then replacing $\mathcal{U}(x, r)$ in $(22)$ with

$$
\mathcal{U}(x, r)=\kappa\left(\frac{V_{1}(x)+V_{2}(x)}{r}\right)+\gamma\left[\sqrt{1+\left(r-r_{\star}\right)^{2}}-1\right]
$$

yields

$$
\begin{aligned}
\overbrace{\mathcal{U}(x, r)} \leq(1-\alpha-\beta-\gamma) W(x, r) \kappa^{\prime} & \left(V_{1}(x)+\frac{V_{2}(x)}{r}\right) \\
& -\gamma \beta \varpi(x, r) \frac{\left(r-r_{\star}\right)^{2}}{\sqrt{1+\left(r-r_{\star}\right)^{2}}} .
\end{aligned}
$$

As a result, the point $\left(0, r_{\star}\right)$ is asymptotically stable, with $\mathbb{R}^{n} \times(R(0),+\infty)$ as basin of attraction.

Remark 5: The existence proof in Proposition 2 relies on the use of universal formulae [25]-[27]. However, in specific examples (see the introductory example) it is possible to design the feedback control and the dynamics of the weighting variable $r$ directly, i.e., without the use of universal formulae.

Remark 6: Propositions 1 and 2 differ in the extra assumption $\ell_{1}^{\prime}\left(V_{1}\right) \geq R \ell_{2}^{\prime}\left(V_{2}\right)$ for the former and $V_{1}+V_{2} / R$ radially unbounded for the latter. Both assumption are trivially satisfied when $V_{1}$ is radially unbounded whereas in general the assumptions of the Proposition 2 on dynamic weighting to hold are (slightly) more restrictive than the necessary conditions for the assumption of the Proposition 1 on static scaling to hold. (Further material can be downloaded at [28]).

\section{CONCLUSION}

The relation between static and dynamic Lyapunov function scaling has been discussed. It has been shown that, under proper technical conditions, the two tools are equivalent. This theoretical, existence, result has been motivated by means of a simple example and has been illustrated on a worked out design problem. Applications of the proposed tool to the stabilization of general cascaded systems (see the preliminary results in [24]) and to output feedback stabilization of system with iISS inverse dynamics are under investigation.

\section{REFERENCES}

[1] L. Praly, D. Carnevale, and A. Astolfi, "Dynamic vs static scaling: An existence result," in IFAC Symposium on Nonlinear Control System Design, Bologna, IT, 2010, pp. 1075-1080.

[2] J. Carr, Applications of Center Manifold Theory. New York: Springer-Verlag, 1981.

[3] H. Khalil, Nonlinear Systems, 3rd ed. Upper Saddle River, NJ: Prentice-Hall, 2002.

[4] E. Sontag and A. Teel, "Changing supply functions in input/state stable systems," IEEE Trans. Automat. Control, vol. 40, pp. 1476-1478, 1995.

[5] Z.-P. Jiang, I. Mareels, and Y. Wang, "A Lyapunov formulation of the nonlinear small-gain theorem for interconnected ISS systems," Automatica, vol. 32, no. 8, pp. 1211-1215, 1996.

[6] D. Angeli and A. Astolfi, "A tight small gain theorem for not necessarily ISS systems," Syst. Control Lett., vol. 56, pp. 87-91, 2007.

[7] H. Ito, "State-dependent scaling problems and stability of interconnected iISS and ISS systems," IEEE Trans. Automat. Control, vol. 51, no. 11 , pp. $1626-1643$, Oct. 2006.

[8] H. Ito and Z.-P. Jiang, "Necessary and sufficient small gain conditions for integral input-to-state stable systems: A Lyapunov perspective," IEEE Trans. Automat. Control, vol. 54, no. 11, pp. 2389-2404, Oct. 2009.

[9] H. Ito, "A Lyapunov approach to cascade interconnection of integral input-to-state stable systems," IEEE Trans. Automat. Control, vol. 55, no. 3, pp. 702-708, Mar. 2010.

[10] S. Dashkovskiy, B. Rüffer, and F. Wirth, "Small gain theorems for large scale systems and construction of ISS lyapunov functions," SIAM J. Control and Optimiz., vol. 48, pp. 4089-4118, 2010.

[11] F. Mazenc and L. Praly, "Adding integrations, saturated controls, and stabilization for feedforward systems," IEEE Trans. Automat. Control, vol. 41, no. 11, pp. 1559-1578, Nov. 1996.

[12] M. Jankovic, R. Sepulchre, and P. Kokotovic, "Constructive Lyapunov stabilization of nonlinear cascade systems," IEEE Trans. Automat. Control, vol. 41, no. 12, pp. 1723-1735, Dec. 1996.

[13] M. Krstic, I. Kanellakopoulos, and P. Kokotovic, Nonlinear and Adaptive Control Design. New York: Wiley, 1995.

[14] Z.-P. Jiang, "A combined backstepping and small-gain approach to adaptive output feedback control," Automatica, vol. 35, no. 6, pp. $1131-1139,1999$.

[15] A. Astolfi, D. Karagiannis, and R. Ortega, Nonlinear and Adaptive Control with Applications. London, U.K.: Springer-Verlag, 2008.

[16] D. Karagianis, M. Sassano, and A. Astolfi, "Dynamic scaling and observer design with application to adaptive control," Automatica, vol. 45, pp. 2883-2889, 2009.

[17] G. Kaliora, A. Astolfi, and L. Praly, "Norm estimators and global output feedback stabilization of nonlinear systems with iss inverse dynamics," IEEE Trans. Automat. Control, vol. 51, no. 3, pp. 493-498, Mar. 2006.

[18] E. Sontag and Y. Wang, "Output-to-state stability and detectability of nonlinear systems," Syst. \& Control Lett., vol. 29, pp. 279-290, 1997.

[19] Z.-P. Jiang and L. Praly, "Preliminary results about Lagrange stability in adaptive nonlinear regulation," Int. J. Adaptive Control and Signal Process., vol. 6, no. 4, pp. 285-307, 1992.

[20] L. Praly and Y. Wang, "Stabilization in spite of matched unmodelled dynamics and an equivalent definition of input-tostate stability," Math. Contr. Sign. Sys., vol. 9, pp. 1-33, 1996. 
[21] A. Astolfi and L. Praly, "Global complete observability and output-tostate stability imply the existence of a globally convergent observer," Mathemat. Control, Signals, and Syst., vol. 18, no. 1, pp. 32-65, 2006

[22] L. Praly, "Asymptotic stabilization via output feedback for lower triangular systems with output dependent incremental rate," IEEE Trans. Automat. Control, vol. 48, no. 6, pp. 1103-1108, 2003.

[23] V. Andrieu, L. Praly, and A. Astolfi, "Asymptotic tracking of a reference trajectory by output-feedback for a class of non-linear systems," Syst. Control Lett., vol. 58, pp. 652-663, 2009.

[24] D. Carnevale and A. Astolfi, "Integrator forwarding without PDEs," in Proc. 48th Conf. Decision and Control, Shanghai, China, 2009, pp. $33-38$.

[25] E. Sontag, "A "universal" construction of Artstein's theorem on nonlinear stabilization," Syst. Control Lett., vol. 13, pp. 117-123, 1989.

[26] R. Freeman and P. Kokotovic, "Inverse optimality in robust stabilization," SIAM J. Control and Optimiz., pp. 1365-1391, 1996.

[27] A. Bacciotti, "Local stabilizability of nonlinear control systems," in Series on Advances in Mathematics for Applied Sciences. Singapore: World Scientific, 1991.

[28] Appendix: Dynamic Versus Static Weighting of Lyapunov Functions [Online]. Available: http://control.disp.uniroma2.it/carnevale/ archivio/Downloads/DynamicWeightingApp.pdf additional material

\section{Stochastic Integration Filter}

Jindřich Duník, Ondřej Straka, and Miroslav Šimandl

\begin{abstract}
The technical note deals with state estimation of nonlinear stochastic dynamic systems. Traditional filters providing local estimates of the state, such as the extended Kalman filter, unscented Kalman filter, or the cubature Kalman filter, are based on computationally efficient but approximate integral evaluations. On the other hand, the Monte Carlo based Kalman filter takes an advantage of asymptotically exact integral evaluations but at the expense of substantial computational demands. The aim of the technical note is to propose a new local filter that utilises stochastic integration methods providing the asymptotically exact integral evaluation with computational complexity similar to the traditional filters. The technical note will demonstrate that the unscented and cubature Kalman filters are special cases of the proposed stochastic integration filter. The proposed filter is illustrated by a numerical example.
\end{abstract}

Index Terms-Bayesian filters, nonlinear filtering, state estimation, stochastic systems.

\section{INTRODUCTION}

The problem of recursive state estimation of discrete-time stochastic dynamic systems from noisy or incomplete measured data has been a subject of considerable research interest for the last several decades.

The general solution to the estimation problem, based on the Bayesian approach, is given by the Bayesian recursive relations (BRRs) for computation of probability density functions (pdfs) of the state conditioned by the measurements. These pdfs provide a full description of the immeasurable state. The closed form solution to the

Manuscript received June 07, 2011; revised March 26, 2012; accepted October 16, 2012. Date of publication April 16, 2013; date of current version May 20, 2013. This work was supported by the Czech Science Foundation, project GACR P103/11/1353. Recommended by Associate Editor M. Prandini.

The authors are with the Department of Cybernetics, Faculty of Applied Sciences, University of West Bohemia, Pilsen 306 14, Czech Republic (e-mail addresses: dunikj@kky.zcu.cz; straka30@kky.zcu.cz; simandl@kky.zcu.cz).

Color versions of one or more of the figures in this technical note are available online at http://ieeexplore.ieee.org.

Digital Object Identifier 10.1109/TAC.2013.2258494
BRRs is available only for a few special cases [1], e.g., for a linear Gaussian system which leads to the well-known Kalman filter (KF). In other cases, it is necessary to apply some approximative methods. These methods can be divided into two groups: local and global methods [2].

The global methods are based on a certain type of approximation of the BRRs and generate the conditional pdf of the state. The global methods are represented by e.g., the particle filter (PF) [3], the pointmass method [4], the Gaussian sum method or the ensemble Kalman filter (EnKF) [5].

The local methods are based on an approximation of a system description so that the KF design technique can be used for the BRRs solution, i.e., the conditional mean and covariance matrix are computed instead of the conditional pdf. This rough approximation of the model and a posteriori estimates induces local validity of the estimates and consequently impossibility to generally ensure convergence of the local filter (LF) estimates. On the other hand, the advantage of the local methods can be found in the simplicity of the BRRs solution. The local methods either approximate the nonlinear functions appearing in the system description or approximate the pdfs representing state estimates. The former group of filters is based on functions' approximation using polynomial expansions, e.g., the Taylor or Stirling expansions [1], [6]-[9]. As an example, the extended Kalman filter (EKF), second order filter or divided difference filters (DDFs) can be mentioned. The latter group approximate the pdfs representing state estimates by a set of deterministically chosen weighted points [8]-[13]. This approach uses the unscented transform (UT) or it can be viewed as an application of deterministic quadrature or cubature integration methods. The unscented Kalman filter (UKF), the Gauss-Hermite filter or the cubature Kalman filter (CKF) exemplify this approach ${ }^{1}$. An alternate way of approximating the pdfs of the state estimate is to use a set of randomly chosen points. This approach is in fact based on Monte Carlo (MC) integration and its application leads to the Monte Carlo Kalman filter (MCKF) [14].

As the local filters follow the structure of the KF algorithm, they can be written in a unified framework [9]. The approximation based on the polynomial expansions, UT or deterministic integration methods usually leads to approximate integral evaluation only, but with low computational complexity. On the other hand, the MC integration allows asymptotically exact integral evaluation. Thus it leads to significantly better filter performance in terms of estimation quality. However, it is connected with a significant increase of computational demands.

The goal of the technical note is to propose a novel more accurate and computationally efficient LF based on stochastic integration methods. The stochastic integration methods provide an asymptotically exact integral evaluation with convergence faster than the MC integration; thus with significantly lower computational costs.

The technical note is organised as follows. System specification and problem statement is given in Section II. The stochastic integration methods and the novel stochastic integration filter are introduced and analysed in Section III. In Section IV, a numerical illustration of the proposed filter is given and concluding remarks are drawn in Section V.

\section{System Description AND Problem Statement}

In this technical note, the discrete-time nonlinear stochastic system

$$
\begin{aligned}
\mathbf{x}_{k+1} & =\mathbf{f}_{k}\left(\mathbf{x}_{k}\right)+\mathbf{w}_{k}, \quad k=0,1,2, \ldots, \\
\mathbf{z}_{k} & =\mathbf{h}_{k}\left(\mathbf{x}_{k}\right)+\mathbf{v}_{k}, \quad k=0,1,2, \ldots
\end{aligned}
$$

${ }^{1}$ It is notable that the UKF and the DDFs (and their variants) can be viewed as one class of filters, namely derivative-free Kalman filters [9], although they are based on different ideas. 